\title{
False-Positive Findings on Dopamine Transporter SPECT Due to Therapeutic Dextroamphetamine and Amphetamine
}

\author{
Joseph A. Frankl ${ }^{1}$, Sudeshna Bose ${ }^{2}$, and Phillip H. Kuo ${ }^{3,4}$ \\ ${ }^{1}$ College of Medicine, University of Arizona, Tucson, Arizona; ${ }^{2}$ Department of Neurology, University of Arizona, Tucson, Arizona; \\ ${ }^{3}$ Department of Medical Imaging, University of Arizona, Tucson, Arizona; and ${ }^{4}$ Departments of Medicine and Biomedical \\ Engineering, University of Arizona, Tucson, Arizona
}

\begin{abstract}
Dopamine transporter SPECT is an accurate adjunct to clinical evaluation for Parkinson disease when the diagnosis is difficult. Dopaminergic medications may significantly affect dopamine transporter availability and, thus, uptake of dopamine transporter tracers. A patient had a false-positive dopamine transporter SPECT result while she was taking dextroamphetamine and amphetamine for attention-deficit hyperactivity disorder. The SPECT findings normalized after amphetamine therapy was withheld. An accurate medication history combined with knowledge of drugs that interfere with dopamine transporter imaging is critical to ensure accuracy.
\end{abstract}

Key Words: dopamine transporter; single-photon positron emission tomography; ioflupane; Parkinson disease; amphetamine

J Nucl Med Technol 2018; 46:149-150

DOI: 10.2967/jnmt.117.201558

\section{D}

opamine transporter localization with ${ }^{123}$ I-ioflupane and SPECT is an accurate and well-established test to differentiate Parkinson disease from similar clinical entities not associated with presynaptic neuronal dysfunction (1). However, the images can be degraded by positioning artifacts and dopaminergic drugs (1-3). We present a case in which dopamine transporter SPECT showed findings that were suggestive of Parkinson disease while the patient was taking dextroamphetamine and amphetamine for attention-deficit hyperactivity disorder and then were normal after amphetamines were withheld. Institutional review board approval was not required for this case report.

\section{CASE REPORT}

A 60-y-old woman with bipolar disorder treated with valproate and ziprasidone, and attention-deficit hyperactivity disorder treated with dextroamphetamine and amphetamine $(15 \mathrm{mg} / \mathrm{d})$, presented with a 2-mo history of progressive

Received Aug. 31, 2017; revision accepted Oct. 30, 2017.

For correspondence or reprints contact: Joseph A. Frankl, University of Arizona College of Medicine, 1501 N. Campbell Ave., Tucson, AZ 85724.

E-mail: jfrankl@email.arizona.edu

Published online Dec. 22, 2017.

COPYRIGHT (c) 2018 by the Society of Nuclear Medicine and Molecular Imaging. tremors and instability. The patient's neurologist started her on carbidopa and levodopa and ordered a dopamine transporter SPECT scan, and her psychiatrist decreased her valproate dosage. The scan, obtained while the patient was taking dextroamphetamine and amphetamine, were interpreted as showing reduced activity in the left putamen, consistent with Parkinson disease (Fig. 1).

Although the patient's symptoms had improved 1 mo after the change in medication, significant residual symptoms remained. Discontinuation of the ziprasidone led to further improvement. Because the symptoms were not responding as would be expected for typical Parkinson disease, and especially because the results of the dopamine transporter imaging were only mildly abnormal, the neurologist and the nuclear medicine physician together reviewed the patient's chart for potentially interfering medications. The physicians agreed to repeat the imaging after the dextroamphetamine and amphetamine had been withheld for $7 \mathrm{wk}$, and the results were normal (Fig. 2). Therefore, drug-induced parkinsonism was considered most likely.

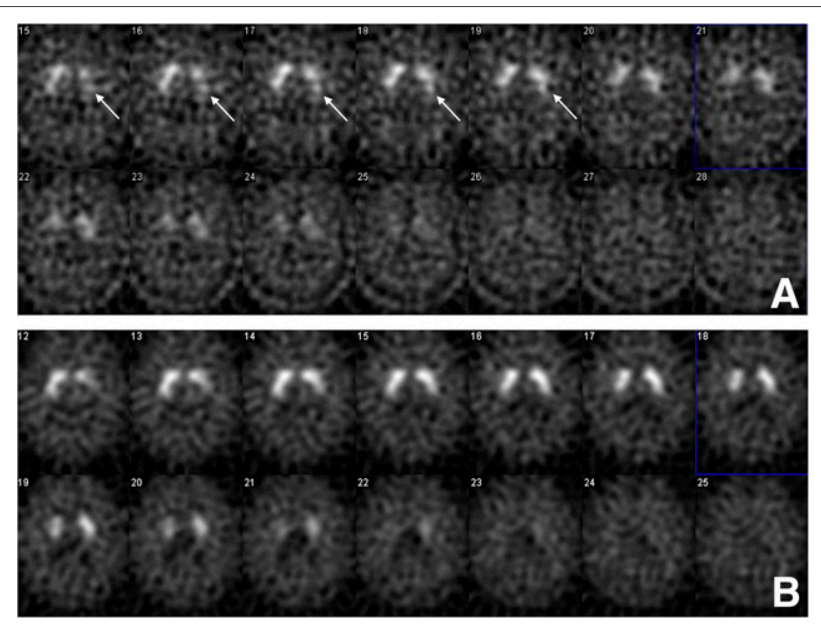

FIGURE 1. (A) Decreased activity in left putamen (arrows) on dopamine transporter SPECT images obtained while patient was receiving dextroamphetamine and amphetamine supports diagnosis of Parkinson disease. (B) Typical normal dopamine transporter SPECT findings using same camera and reconstruction protocol. 


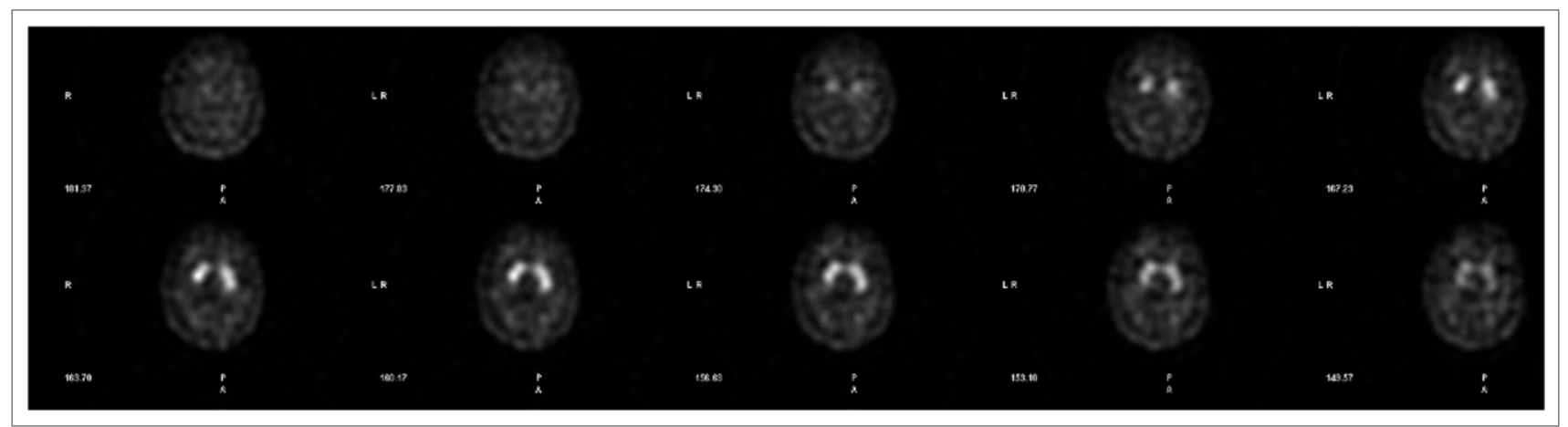

FIGURE 2. Dopamine transporter SPECT images obtained on different camera showed normalization after 7 wk without dextroamphetamine and amphetamine.

\section{DISCUSSION}

The patient's initial dopamine transporter SPECT study, obtained while she was taking amphetamines for attentiondeficit hyperactivity disorder, was positive for Parkinson disease, but the repeated study, obtained after amphetamines had been withheld for $7 \mathrm{wk}$, was negative for Parkinson disease. This case demonstrates that therapeutic doses of amphetamines can significantly alter dopamine transporter imaging. Our findings are consistent with animal models showing that methylphenidate-which, like dextroamphetamine and amphetamine, reduces dopamine transporter availability-decreases the dopamine transporter SPECT signal (4).

For patients taking amphetamines, a 1-wk washout period has been recommended before dopamine transporter SPECT (1). However, even after $2 \mathrm{wk}$ of abstinence, recreational users of dextroamphetamine have been shown to have a lower striatal SPECT signal than healthy controls (5). Although one case is not sufficient to determine an optimal washout period, $7 \mathrm{wk}$ was used here for normalization of dopamine transporter imaging.

\section{CONCLUSION}

An accurate medical history and knowledge of medications that interfere with dopamine transporter imaging are critical to ensure that drugs are properly withheld beforehand.

\section{DISCLOSURE}

Phillip H. Kuo has consulted for and received grants from GE Healthcare. No other potential conflict of interest relevant to this article was reported.

\section{REFERENCES}

1. Kägi G, Bhatia KP, Tolosa E. The role of DAT-SPECT in movement disorders. J Neurol Neurosurg Psychiatry. 2010;81:5-12.

2. Covington MF, McMillan NA, Avery RJ, Kuo PH. The semicolon sign: dopamine transporter imaging artifact from head tilt. J Nucl Med Technol. 2013;41:105-107.

3. Janicek AK, Avery RJ, Kuo PH. The pinwheel sign: artifact from head rotation during SPECT acquisition for dopamine transporter imaging. J Nucl Med Technol. 2014;42:75-76.

4. Nikolaus S, Wirrwar A, Antke C, et al. Quantitation of dopamine transporter blockade by methylphenidate: first in vivo investigation using [ $\left.{ }^{123} \mathrm{I}\right] \mathrm{FP}-\mathrm{CIT}$ and a dedicated small animal SPECT. Eur J Nucl Med Mol Imaging. 2005;32:308-313.

5. Schouw MLJ, Caan MWA, Geurts HM, et al. Monoaminergic dysfunction in recreational users of dexamphetamine. Eur Neuropsychopharmacol. 2013;23:1491-1502.

\section{Erratum}

In the March issue, we accidentally reprinted a JRCNMT report from a previous issue ( $\mathrm{J}$ Nucl Med Technol. 2018;46:9A-14A). The correct report appears in this issue. We regret the error. 